\title{
An introduction to 2-fuzzy $n$-normed linear spaces and a new perspective to the Mazur-Ulam problem
}

\author{
Choonkil Park ${ }^{1}$ and Cihangir Alaca ${ }^{2 *}$
}

\author{
* Correspondence: \\ cihangiralaca@yahoo.com.tr \\ 2Department of Mathematics, \\ Faculty of Science and Arts, Celal \\ Bayar University, 45140 Manisa, \\ Turkey \\ Full list of author information is \\ available at the end of the article
}

\begin{abstract}
The purpose of this article is to introduce the concept of 2-fuzzy $n$-normed linear space or fuzzy $n$-normed linear space of the set of all fuzzy sets of a non-empty set. We define the concepts of $n$-isometry, $n$-collinearity $n$-Lipschitz mapping in this space. Also, we generalize the Mazur-Ulam theorem, that is, when $X$ is a 2-fuzzy $n$ normed linear space or $\mathfrak{I}(X)$ is a fuzzy $n$-normed linear space, the Mazur-Ulam theorem holds. Moreover, it is shown that each n-isometry in 2-fuzzy n-normed linear spaces is affine.

Mathematics Subject Classification (2010): 03E72; 46B20; 51M25; 46B04; 46540.

Keywords: Mazur-Ulam theorem, $a$-n-norm, 2-fuzzy n-normed linear spaces, $n$-isometry, n-Lipschitz mapping.
\end{abstract}

\section{Introduction}

A satisfactory theory of 2-norms and n-norms on a linear space has been introduced and developed by Gähler [1,2]. Following Misiak [3], Kim and Cho [4], and Malčeski [5] developed the theory of $n$-normed space. In [6], Gunawan and Mashadi gave a simple way to derive an $(n-1)$-norm from the $n$-norms and realized that any $n$-normed space is an $(n-1)$-normed space. Different authors introduced the definitions of fuzzy norms on a linear space. Cheng and Mordeson [7] and Bag and Samanta [8] introduced a concept of fuzzy norm on a linear space. The concept of fuzzy $n$-normed linear spaces has been studied by many authors (see [4,9]).

Recently, Somasundaram and Beaula [10] introduced the concept of 2-fuzzy 2normed linear space or fuzzy 2-normed linear space of the set of all fuzzy sets of a set. The authors gave the notion of $\alpha$-2-norm on a linear space corresponding to the 2fuzzy 2-norm by using some ideas of Bag and Samanta [8] and also gave some fundamental properties of this space.

In 1932, Mazur and Ulam [11] proved the following theorem.

Mazur-Ulam Theorem. Every isometry of a real normed linear space onto a real normed linear space is a linear mapping up to translation.

Baker [12] showed an isometry from a real normed linear space into a strictly convex real normed linear space is affine. Also, Jian [13] investigated the generalizations of the Mazur-Ulam theorem in $F^{*}$-spaces. Rassias and Wagner [14] described all volume preserving mappings from a real finite dimensional vector space into itself and Väisälä

(c) 2012 Park and Alaca; licensee Springer. This is an Open Access article distributed under the terms of the Creative Commons Attribution License (http://creativecommons.org/licenses/by/2.0), which permits unrestricted use, distribution, and reproduction in any medium, provided the original work is properly cited. 
[15] gave a short and simple proof of the Mazur-Ulam theorem. Chu [16] proved that the Mazur-Ulam theorem holds when $\mathrm{X}$ is a linear 2-normed space. Chu et al. [17] generalized the Mazur-Ulam theorem when $\mathrm{X}$ is a linear $n$-normed space, that is, the Mazur-Ulam theorem holds, when the $n$-isometry mapped to a linear $n$-normed space is affine. They also obtain extensions of Rassias and Šemrl's theorem [18]. Moslehian and Sadeghi [19] investigated the Mazur-Ulam theorem in non-archimedean spaces. Choy et al. [20] proved the Mazur-Ulam theorem for the interior preserving mappings in linear 2-normed spaces. They also proved the theorem on non-Archimedean 2normed spaces over a linear ordered non-Archimedean field without the strict convexity assumption. Choy and $\mathrm{Ku}$ [21] proved that the barycenter of triangle carries the barycenter of corresponding triangle. They showed the Mazur-Ulam problem on nonArchimedean 2-normed spaces using the above statement. Xiaoyun and Meimei [22] introduced the concept of weak $n$-isometry and then they got under some conditions, a weak $n$-isometry is also an $n$-isometry. Cobzaş [23] gave some results of the MazurUlam theorem for the probabilistic normed spaces as defined by Alsina et al. [24]. Cho et al. [25] investigated the Mazur-Ulam theorem on probabilistic 2-normed spaces. Alaca [26] introduced the concepts of 2-isometry, collinearity, 2-Lipschitz mapping in 2-fuzzy 2-normed linear spaces. Also, he gave a new generalization of the Mazur-Ulam theorem when $X$ is a 2-fuzzy 2-normed linear space or $\mathfrak{I}(X)$ is a fuzzy 2-normed linear space. Kang et al. [27] proved that the Mazur-Ulam theorem holds under some conditions in non-Archimedean fuzzy normed space. Kubzdela [28] gave some new results for isometries, Mazur-Ulam theorem and Aleksandrov problem in the framework of non-Archimedean normed spaces. The Mazur-Ulam theorem has been extensively studied by many authors (see $[29,30])$.

In the present article, we introduce the concept of 2-fuzzy $n$-normed linear space or fuzzy $n$-normed linear space of the set of all fuzzy sets of a non-empty set. We define the concepts of $n$-isometry, $n$-collinearity, $n$-Lipschitz mapping in this space. Also, we generalize the Mazur-Ulam theorem, that is, when $X$ is a 2 -fuzzy $n$-normed linear space or $\mathfrak{I}(X)$ is a fuzzy $n$-normed linear space, the Mazur-Ulam theorem holds. It is moreover shown that each $n$-isometry in 2 -fuzzy $n$-normed linear spaces is affine.

\section{Preliminaries}

Definition 2.1([31]) Let $n \in \mathbb{N}$ and let $X$ be a real vector space of dimension $d \geq n$.

(Here we allow $d$ to be infinite.) A real-valued function $\|\bullet, \ldots, \bullet\|$ on $\underbrace{X \times \cdots \times X}_{n}$ satisfying the following properties

(1) $\left\|x_{1}, x_{2}, \ldots, x_{n}\right\|=0$ if and only if $x_{1}, x_{2}, \ldots, x_{n}$ are linearly dependent,

(2) $\left\|x_{1}, x_{2}, \ldots, x_{n}\right\|$ is invariant under any permutation,

(3) $\left\|x_{1}, x_{2}, \ldots, \alpha x_{n}\right\|=|\alpha|\left\|x_{1}, x_{2}, \ldots, x_{n}\right\|$ for any $\alpha \in \mathbb{R}$,

(4) $\left\|x_{1}, x_{2}, \ldots, x_{n-1}, y+z\right\| \leq\left\|x_{1}, x_{2}, \ldots, x_{n-1}, y\right\|+\left\|x_{1}, x_{2}, \ldots, x_{n-1}, z\right\|$, is called an $n$ norm on $X$ and the pair $(X,\|\bullet, \ldots, \bullet\|)$ is called an $n$-normed linear space.

Definition 2.2 [9] Let $X$ be a linear space over $S$ (field of real or complex numbers). A fuzzy subset $N$ of $X^{n} \times \mathbb{R}$ ( $\mathbb{R}$, the set of real numbers) is called a fuzzy $n$-norm on $X$ 
if and only if:

(N1) For all $t \in \mathbb{R}$ with $t \leq 0, N\left(x_{1}, x_{2}, \ldots, x_{n}, t\right)=0$,

(N2) For all $t \in \mathbb{R}$ with $t>0, N\left(x_{1}, x_{2}, \ldots, x_{n}, t\right)=1$ if and only if $x_{1}, x_{2}, \ldots, x_{n}$ are linearly dependent,

(N3) $N\left(x_{1}, x_{2}, \ldots, x_{n}, t\right)$ is invariant under any permutation of $x_{1}, x_{2}, \ldots, x_{n}$,

(N4) For all $t \in \mathbb{R}$ with $t>0, N\left(x_{1}, x_{2}, \ldots, \lambda x_{n}, t\right)=N\left(x_{1}, x_{2}, \ldots, x_{n}, \frac{t}{\lambda}\right)$, if $\lambda \neq 0$,

$\lambda \in S$,

(N5) For all $s, t \in \mathbb{R}$

$N\left(x_{1}, x_{2}, \ldots, x_{n}+x_{n}^{\prime} s+t\right) \geq \min \left\{N\left(x_{1}, x_{2}, \ldots, x_{n}, s\right), N\left(x_{1}, x_{2}, \ldots, x_{n}^{\prime}, t\right)\right\}$,

(N6) $N\left(x_{1}, x_{2}, \ldots, x_{n}, t\right)$ is a non-decreasing function of $t \in \mathbb{R}$ and $\lim _{t \rightarrow \infty} N\left(x_{1}, x_{2}, \ldots, x_{n}, t\right)=1$.

Then $(X, N)$ is called a fuzzy $n$-normed linear space or in short $f$ - $n$-NLS.

Theorem 2.1 [9] Let $(X, N)$ be an $f$-n-NLS. Assume that

(N7) $N\left(x_{1}, x_{2}, \ldots, x_{n}, t\right)>0$ for all $t>0$ implies that $x_{1}, x_{2}, \ldots, x_{n}$ are linearly dependent.

Define

$$
\left\|x_{1}, x_{2}, \ldots, x_{n}\right\|_{\alpha}=\inf \left\{t: N\left(x_{1}, x_{2}, \ldots x_{n}, t\right) \geq \alpha, \alpha \in(0,1)\right\} .
$$

Then $\left\{\|\bullet, \bullet, \ldots, \bullet\|_{\alpha}: \alpha \in(0,1)\right\}$ is an ascending family of $n$-norms on $X$.

We call these $n$-norms as $\alpha$-n-norms on $X$ corresponding to the fuzzy $n$-norm on $X$.

Definition 2.3 Let $X$ be any non-empty set and $\mathfrak{I}(X)$ the set of all fuzzy sets on $X$. For $U, V \in \Im(X)$ and $\lambda \in S$ the field of real numbers, define

$$
U+V=\{(x+y, v \wedge \mu):(x, v) \in U,(y, \mu) \in V\}
$$

and $\lambda U=\{(\lambda x, v):(x, v) \in U\}$

Definition 2.4 A fuzzy linear space $\widehat{X}=X \times(0,1]$ over the number field $S$, where the addition and scalar multiplication operation on $X$ are defined by $(x, v)+(y, \mu)=(x$ $+y, v \wedge \mu), \lambda(x, v)=(\lambda x, v)$ is a fuzzy normed space if to every $(x, v) \in \widehat{X}$ there is associated a non-negative real number, $\|(x, v)\|$, called the fuzzy norm of $(x, v)$, in such away that

(i) $\|(x, v)\|=0$ iff $x=0$ the zero element of $X, v \in(0,1]$,

(ii) $\|\lambda(x, v)\|=|\lambda|\|(x, v)\|$ for all $(x, v) \in \widehat{X}$ and all $\lambda \in S$,

(iii) $\|(x, v)+(y, \mu)\| \leq\|(x, v \wedge \mu)\|+\|(y, v \wedge \mu)\|$ for all $(x, v),(y, \mu) \in \widehat{X}$,

(iv) $\left\|\left(x, v_{t} v_{t}\right)\right\|=\Lambda_{t}\left\|\left(x, v_{t}\right)\right\|$ for all $v_{t} \in(0,1]$. 


\section{2-fuzzy $\boldsymbol{n}$-normed linear spaces}

In this section, we define the concepts of 2 -fuzzy $n$-normed linear spaces and $\alpha-n$ norms on the set of all fuzzy sets of a non-empty set.

Definition 3.1 Let $X$ be a non-empty and $\Im(X)$ be the set of all fuzzy sets in $X$. If $f \in$ $\mathfrak{I}(X)$ then $f=\{(x, \mu): x \in X$ and $\mu \in(0,1]\}$. Clearly $f$ is bounded function for $|f(x)| \leq 1$. Let $S$ be the space of real numbers, then $\mathfrak{S}(X)$ is a linear space over the field $S$ where the addition and scalar multiplication are defined by

$$
f+g=\{(x, \mu)+(y, \eta)\}=\{(x+y, \mu \wedge \eta):(x, \mu) \in f \text { and }(y, \eta) \in g\}
$$

and

$$
\lambda f=\{(\lambda x, \mu):(x, \mu) \in f\}
$$

where $\lambda \in S$.

The linear space $\mathfrak{I}(X)$ is said to be normed linear space if, for every $f \in \mathfrak{I}(X)$, there exists an associated non-negative real number $\|f\|$ (called the norm of $f$ ) which satisfies

(i) $\|f\|=0$ if and only if $f=0$. For

$$
\begin{aligned}
\|f\| & =0 \\
& \Leftrightarrow\{\|(x, \mu)\|:(x, \mu) \in f\}=0 \\
& \Leftrightarrow x=0, \mu \in(0,1] \Leftrightarrow f=0 .
\end{aligned}
$$

(ii) $\|\lambda f\|=|\lambda|\|f\|, \lambda \in S$. For

$$
\begin{aligned}
\|\lambda f\| & =\{\|\lambda(x, \mu)\|:(x, \mu) \in f, \lambda \in S\} \\
& =\{|\lambda|\|(x, \mu)\|:(x, \mu) \in f\}=|\lambda|\|f\| .
\end{aligned}
$$

(iii) $\|f+g\| \leq\|f\|+\|g\|$ for every $f, g \in \mathfrak{S}(X)$. For

$$
\begin{aligned}
\|f+g\| & =\{\|(x, \mu)+(y, \eta)\|: x, y \in X, \mu, \eta \in(0,1]\} \\
& =\{\|(x+\gamma),(\mu \wedge \eta)\|: x, y \in X, \mu, \eta \in(0,1]\} \\
& =\{\|(x, \mu \wedge \eta)\|+\|(y, \mu \wedge \eta)\|:(x, \mu) \in f,(y, \eta) \in g\} \\
& =\|f\|+\|g\| .
\end{aligned}
$$

Then $(\mathfrak{S}(X),\|\bullet\|)$ is a normed linear space.

Definition 3.2 A 2-fuzzy set on $X$ is a fuzzy set on $\mathfrak{I}(X)$.

Definition 3.3 Let $X$ be a real vector space of dimension $d \geq n(n \in \mathbb{N})$ and $\mathfrak{S}(X)$ be the set of all fuzzy sets in $X$. Here we allow $d$ to be infinite. Assume that a $[0,1]$ valued function $\|\bullet, \ldots, \bullet\|$ on $\underbrace{\Im(X) \times \cdots \times \Im(X)}_{n}$ satisfies the following properties

(1) $\left\|f_{1}, f_{2}, \ldots, f_{n}\right\|=0$ if and only if $f_{1}, f_{2}, \ldots, f_{n}$ are linearly dependent,

(2) $\left\|f_{1}, f_{2}, \ldots, f_{n}\right\|$ is invariant under any permutation,

(3) $\left\|f_{1}, f_{2}, \ldots, \lambda f_{n}\right\|=|\lambda|\left\|f_{1}, f_{2}, \ldots, f_{n}\right\|$ for any $\lambda \in S$, 
(4) $\left\|f_{1}, f_{2}, \ldots, f_{n-1}, y+z\right\| \leq\left\|f_{1}, f_{2}, \ldots, f_{n-1}, y\right\|+\left\|f_{1}, f_{2}, \ldots, f_{n-1}, z\right\|$.

Then $(\mathfrak{I}(X),\|\bullet, \ldots, \bullet\|)$ is an $n$-normed linear space or $(X,\|\bullet, \ldots, \bullet\|)$ is a 2 -n-normed linear space.

Definition 3.4 Let $\mathfrak{S}(X)$ be a linear space over the real field $S$. A fuzzy subset $N$ of $\underbrace{\Im(X) \times \cdots \times \Im(X)}_{n} \times \mathbb{R}$ is called a 2 -fuzzy $n$-norm on $X$ (or fuzzy $n$-norm on $\mathfrak{I}(X)$ ) if and only if

(2-N1) for all $t \in \mathbb{R}$ with $t \leq 0, N\left(f_{1}, f_{2}, \ldots, f_{n}, t\right)=0$,

(2-N2) for all $t \in \mathbb{R}$ with $t>0, N\left(f_{1}, f_{2}, \ldots, f_{n}, t\right)=1$ if and only if $f_{1}, f_{2}, \ldots, f_{n}$ are linearly dependent,

(2-N3) $N\left(f_{1}, f_{2}, \ldots, f_{n}, t\right)$ is invariant under any permutation of $f_{1}, f_{2}, \ldots, f_{n}$,

(2-N4) for all $t \in \mathbb{R}$ with $t>0, N\left(f_{1}, f_{2}, \ldots, \lambda f_{n}, t\right)=N\left(f_{1}, f_{2}, \ldots, f_{n}, t /|\lambda|\right)$, if $\lambda \neq 0$, $\lambda \in S$,

(2-N5) for all $s, t \in \mathbb{R}$,

$$
N\left(f_{1}, f_{2}, \ldots, f_{n}+f_{n}^{\prime}, s+t\right) \geq \min \left\{N\left(f_{1}, f_{2}, \ldots, f_{n}, s\right), N\left(f_{1}, f_{2}, \ldots, f_{n}^{\prime}, t\right)\right\},
$$

(2-N6) $N\left(f_{1}, f_{2}, \ldots, f_{n}, \cdot\right):(0, \infty) \rightarrow[0,1]$ is continuous,

(2-N7) $\lim _{t \rightarrow \infty} N\left(f_{1}, f_{2}, \ldots, f_{n}, t\right)=1$.

Then $\mathfrak{I}(X), N)$ is a fuzzy $n$-normed linear space or $(X, N)$ is a 2 -fuzzy $n$-normed linear space.

Remark 3.1 In a 2-fuzzy $n$-normed linear space $(X, N), N\left(f_{1}, f_{2}, \ldots, f_{n}, \cdot\right)$ is a nondecreasing function of $\mathbb{R}$ for all $f_{1}, f_{2}, \ldots, f_{n} \in \mathfrak{S}(X)$.

Remark 3.2 From (2-N4) and (2-N5), it follows that in a 2 -fuzzy $n$-normed linear space,

$\begin{array}{llllll}(2-\mathrm{N} 4) & \text { for } & \text { all } & t & \mathrm{R} & \text { with }\end{array}$ $N\left(f_{1}, f_{2}, \ldots, \lambda f_{i}, \ldots, f_{n}, t\right)=N\left(f_{1}, f_{2}, \ldots, f_{i}, \ldots, f_{n}, \frac{t}{|\lambda|}\right)$, if $\lambda \neq 0, \lambda \in S$,

(2-N5) for all $s, t \in \mathbb{R}$,

$$
\begin{aligned}
& N\left(f_{1}, f_{2}, \ldots, f_{i}+f^{\prime}{ }_{i}, \ldots, f_{n}, s+t\right) \\
& \quad \geq \min \left\{N\left(f_{1}, f_{2}, \ldots, f_{i}, \ldots, f_{n}, s\right), N\left(f_{1}, f_{2}, \ldots, f^{\prime}{ }_{i}, \ldots, f_{n}, t\right)\right\} .
\end{aligned}
$$

The following example agrees with our notion of 2-fuzzy $n$-normed linear space.

Example 3.1 Let $(\mathfrak{I}(X),\|\bullet, \bullet, \ldots, \bullet\|)$ be an $n$-normed linear space as in Definition 3.3. Define

$$
N\left(f_{1}, f_{2}, \ldots, f_{n}, t\right)=\left\{\begin{array}{c}
\frac{t}{t+\left\|f_{1}, f_{2}, \ldots, f_{n}\right\|} \text { if } t>0, t \in \mathbb{R} \\
\text { Oif } t \leq 0
\end{array}\right.
$$

for all $\left(f_{1}, f_{2}, \ldots, f_{n}\right) \in \underbrace{\Im(X) \times \cdots \times \Im(X)}_{n}$. Then $(X, N)$ is a 2-fuzzy $n$-normed linear space.

Solution. (2-N1) For all $t \in \mathbb{R}$ with $t \leq 0$, by definition, we have $N\left(f_{1}, f_{2}, \ldots, f_{n}, t\right)=0$. 
(2-N2) For all $t \in \mathbb{R}$ with $t>0$,

$$
\begin{aligned}
N\left(f_{1}, f_{2}, \ldots, f_{n}, t\right) & =1 \Leftrightarrow \frac{t}{t+\left\|f_{1}, f_{2}, \ldots, f_{n}\right\|}=1 \\
& \Leftrightarrow t=t+\left\|f_{1}, f_{2}, \ldots, f_{n}\right\| \\
& \Leftrightarrow\left\|f_{1}, f_{2}, \ldots, f_{n}\right\|=0 \\
& \Leftrightarrow f_{1}, f_{2}, \ldots, f_{n} \text { are linearly dependent. }
\end{aligned}
$$

(2-N3) For all $t \in \mathbb{R}$ with $t>0$,

$$
\begin{aligned}
N\left(f_{1}, f_{2}, \ldots, f_{n}, t\right) & =\frac{t}{t+\left\|f_{1}, f_{2}, \ldots, f_{n}\right\|}=\frac{t}{t+\left\|f_{1}, f_{2}, \ldots, f_{n}, f_{n-1}\right\|} \\
& =N\left(f_{1}, f_{2}, \ldots, f_{n}, f_{n-1}, t\right)=\cdots .
\end{aligned}
$$

(2-N4) For all $t \in \mathbb{R}$ with $t>0$ and $\lambda \in F, \lambda \neq 0$,

$$
\begin{aligned}
N\left(f_{1}, f_{2}, \ldots, f_{n}, t /|\lambda|\right) & =\frac{t /|\lambda|}{t /|\lambda|+|| f_{1}, f_{2}, \ldots, f_{n} \|} \\
& =\frac{t /|\lambda|}{\left(t+|\lambda||| f_{1}, f_{2}, \ldots, f_{n} \|\right) /|\lambda|} \\
& =\frac{t}{t+|\lambda||| f_{1}, f_{2}, \ldots, f_{n} \|} \\
& =\frac{t}{\left(t+|| f_{1}, f_{2}, \ldots, \lambda f_{n} \|\right.}=N\left(f_{1}, f_{2}, \ldots, \lambda f_{n}, t\right) .
\end{aligned}
$$

(2-N5) We have to prove

$$
N\left(f_{1}, f_{2}, \ldots, f_{n}+f_{n}^{\prime}, s+t\right) \geq \min \left\{f\left(x_{1}, f_{2}, \ldots, f_{n}, s\right), N\left(f_{1}, f_{2}, \ldots, f_{n}^{\prime}, t\right)\right\} .
$$

(i) $s+t<0$,

(ii) $s=t=0$,

(iii) $s+t>0$; $s>0, t<0$; $s<0, t>0$, then the above relation is obvious. If

(iv) $s>0, t>0, s+t>0$, then

$$
N\left(f_{1}, f_{2}, \ldots, f_{n}+f_{n^{\prime}}^{\prime} s+t=\frac{s+t}{s+t+\left\|f_{1}, f_{2}, \ldots, f_{n}+f^{\prime}{ }_{n}\right\|} .\right.
$$

If

$$
\begin{aligned}
& \frac{s}{s+\left\|f_{1}, f_{2}, \ldots, f_{n}\right\|} \geq \frac{t}{t+\left\|f_{1}, f_{2}, \ldots, f_{n}^{\prime}\right\|} \Rightarrow \frac{\left\|f_{1}, f_{2}, \ldots, f_{n}\right\|}{s} \leq \frac{\left\|x_{1}, x_{2}, \ldots, x_{n}^{\prime}\right\|}{t} \\
& \Rightarrow \frac{\left\|f_{1}, f_{2}, \ldots, f_{n}\right\|}{s}+\frac{\left\|f_{1}, f_{2}, \ldots, f^{\prime}{ }_{n}\right\|}{s} \leq \frac{\left\|f_{1}, f_{2}, \ldots, f_{n}^{\prime}\right\|}{t} \\
& +\frac{\left\|f_{1}, f_{2}, \ldots, f_{n}^{\prime}\right\|}{s} \\
& \Rightarrow \frac{\left\|f_{1}, f_{2}, \ldots, f_{n}+f_{n}^{\prime}\right\|}{s} \leq\left(\frac{s+t}{s \cdot t}\right)\left\|f_{1}, f_{2}, \ldots, f^{\prime}{ }_{n}\right\| \\
& \Rightarrow \frac{\left\|f_{1}, f_{2}, \ldots, f_{n}+f_{n}^{\prime}\right\|}{s+t} \leq \frac{\left\|f_{1}, f_{2}, \ldots, f_{n}^{\prime}\right\|}{t} \\
& \Rightarrow \frac{s+t+\left\|f_{1}, f_{2}, \ldots, f_{n}+f_{n}^{\prime}\right\|}{s+t} \leq \frac{t+\left\|f_{1}, f_{2}, \ldots, f_{n}^{\prime}\right\|}{t} \\
& \Rightarrow \frac{s+t}{s+t+\left\|f_{1}, f_{2}, \ldots, f_{n}+f_{n}^{\prime}{ }_{n}\right\|} \geq \frac{t}{t+\left\|f_{1}, f_{2}, \ldots, f_{n}^{\prime}\right\|} \\
& \Rightarrow N\left(f_{1}, f_{2}, \ldots, f_{n}+f_{n^{\prime}}^{\prime} s+t\right) \geq N\left(f_{1}, f_{2}, \ldots, f^{\prime}{ }^{\prime}, t\right) \text {. }
\end{aligned}
$$


Similarly, if

$$
\begin{aligned}
& \frac{t}{t+\left\|f_{1}, f_{2}, \ldots, f^{\prime}{ }_{n}\right\|} \geq \frac{s}{s+\left\|f_{1}, f_{2}, \ldots, f_{n}\right\|} \\
& \quad \Rightarrow N\left(f_{1}, f_{2}, \ldots, f_{n}+f^{\prime}{ }^{\prime}, s+t\right) \geq N\left(f_{1}, f_{2}, \ldots, f_{n}, t\right) .
\end{aligned}
$$

Thus

$$
N\left(f_{1}, f_{2}, \ldots, f_{n}+f_{n^{\prime}}^{\prime} s+t\right) \geq \min \left\{N\left(f_{1}, f_{2}, \ldots, f_{n}, s\right), N\left(f_{1}, f_{2}, \ldots, f_{n}^{\prime}, t\right)\right\} .
$$

(2-N6) It is clear that $N\left(f_{1}, f_{2}, \ldots, f_{n}, \cdot\right):(0, \infty) \rightarrow[0,1]$ is continuous.

(2-N7) For all $t \in \mathbb{R}$ with $t>0$,

$$
\begin{aligned}
\lim _{t \rightarrow \infty} N\left(f_{1}, f_{2}, \ldots, f_{n}, t\right) & =\lim _{t \rightarrow \infty} \frac{t}{t+\left\|f_{1}, f_{2}, \ldots, f_{n}\right\|} \\
& =\lim _{t \rightarrow \infty} \frac{t}{t\left(1+(1 / t)\left\|f_{1}, f_{2}, \ldots, f_{n}\right\|\right)}=1,
\end{aligned}
$$

as desired.

As a consequence of Theorem 3.2 in [10], we introduce an interesting notion of ascending family of $\alpha$-n-norms corresponding to the fuzzy $n$-norms in the following theorem.

Theorem 3.1 Let $(\mathfrak{3}(X), N)$ is a fuzzy $n$-normed linear space. Assume that (2-N8) $N\left(f_{1}, f_{2}, \ldots, f_{n}, t\right)>0$ for all $t>0$ implies $f_{1}, f_{2}, \ldots, f_{n}$ are linearly dependent.

Define

$$
\left\|f_{1}, f_{2}, \ldots, f_{n}\right\|_{\alpha}=\inf \left(t: N\left(f_{1}, f_{2}, \ldots, f_{n}, t\right) \geq \alpha, \alpha \in(0,1)\right\} .
$$

Then $\left\{\|\bullet, \bullet, \ldots, \bullet\|_{\alpha}: \alpha \in(0,1)\right\}$ is an ascending family of $n$-norms on $\mathfrak{\Im}(X)$.

These $n$-norms are called $\alpha$ - $n$-norms on $\mathfrak{I}(X)$ corresponding to the 2 -fuzzy $n$-norm on $X$.

Proof. (i) Let $\left\|f_{1}, \ldots, f_{n}\right\|_{\alpha}=0$. This implies that inf $\left\{t: N\left(f_{1}, \ldots, f_{n}, t\right) \geq \alpha\right\}$. Then, $N\left(f_{1}\right.$, $\left.f_{2}, \ldots, f_{n}, t\right) \geq \alpha>0$, for all $t>0, \alpha \in(0,1)$, which implies that $f_{1}, f_{2}, \ldots, f_{n}$ are linearly dependent, by (2-N8).

Conversely, assume $f_{1}, f_{2}, \ldots, f_{n}$ are linearly dependent. This implies that $N\left(f_{1}, f_{2}, \ldots, f_{n}\right.$, $t)=1$ for all $t>0$. For all $\alpha \in(0,1)$, inf $\left\{t: N\left(f_{1}, f_{2}, \ldots, f_{n}, t\right) \geq \alpha\right\}$, which implies that $\left\|f_{1}, f_{2}, \ldots, f_{n}\right\|_{\alpha}=0$.

(ii) Since $N\left(f_{1}, f_{2}, \ldots, f_{n}, t\right)$ is invariant under any permutation, $\left\|f_{1}, f_{2}, \ldots, f_{n}\right\|_{\alpha}=0$ under any permutation.

(iii) If $\lambda \neq 0$, then

$$
\begin{aligned}
\left\|f_{1}, f_{2}, \ldots, \lambda f_{n}\right\|_{\alpha} & =\inf \left\{s: N\left(f_{1}, f_{2}, \ldots, f_{n}, s\right) \geq \alpha\right\} \\
& =\inf \left\{s: N\left(f_{1}, f_{2}, \ldots, f_{n}, \frac{s}{|\lambda|} \geq \alpha\right\} .\right.
\end{aligned}
$$

Let $t=\frac{s}{|\lambda|}$, then

$$
\begin{aligned}
\left\|f_{1}, f_{2}, \ldots, \lambda f_{n}\right\|_{\alpha} & =\inf \left\{|\lambda| t: N\left(f_{1}, f_{2}, \ldots, f_{n}, t\right) \geq \alpha\right\} \\
& =|\lambda| \inf \left\{t: N\left(f_{1}, f_{2}, \ldots, f_{n}, t\right) \geq \alpha\right\}=|\lambda|\left\|f_{1}, f_{2}, \ldots, f_{n}\right\| \alpha .
\end{aligned}
$$


If $\lambda=0$, then

$$
\begin{aligned}
\left\|f_{1}, f_{2}, \ldots, \lambda f_{n}\right\|_{\alpha} & =\left\|f_{1}, f_{2}, \ldots, 0\right\|_{\alpha}=0=0\left\|f_{1}, f_{2}, \ldots, f_{n}\right\| \alpha \\
& =|\lambda|\left\|f_{1}, f_{2}, \ldots, f_{n}\right\| \alpha, \forall \lambda \in S \text { (field). }
\end{aligned}
$$

(iv)

$$
\begin{aligned}
& \left\|f_{1}, f_{2}, \ldots, f_{n}\right\|_{\alpha}+\left\|f_{1}, f_{2}, \ldots, f^{\prime}{ }_{n}\right\| \|_{\alpha} \\
& \quad=\inf \left\{t: N\left(f_{1}, f_{2}, \ldots, f_{n}, t\right) \geq \alpha\right\}+\inf \left(s: N\left(f_{1}, f_{2}, \ldots, f^{\prime}{ }_{n^{\prime}} s\right) \geq \alpha\right\} \\
& \quad=\inf \left\{t+s: N\left(f_{1}, f_{2}, \ldots, f_{n}, t\right) \geq \alpha, N\left(f_{1}, f_{2}, \ldots, f_{n^{\prime}}, s\right) \geq \alpha\right\} \\
& \quad \geq \inf \left\{t+s: N\left(f_{1}, f_{2}, \ldots, f_{n}+f^{\prime}{ }_{n^{\prime}} t+s\right) \geq \alpha\right\}, \\
& \quad \geq \inf \left\{r: N\left(f_{1}, f_{2}, \ldots, f_{n}+f^{\prime}{ }_{n^{\prime}} r\right) \geq \alpha\right\}, r=t+s \\
& =\left\|f_{1}, f_{2}, \ldots, f_{n}+f^{\prime}{ }_{n}\right\|_{\alpha} .
\end{aligned}
$$

Hence

$$
\left\|f_{1}, f_{2}, \ldots, f_{n}+f_{n}^{\prime}\right\|_{\alpha} \leq\left\|f_{1}, f_{2}, \ldots, f_{n}\right\|_{\alpha}+\left\|f_{1}, f_{2}, \ldots, f_{n}^{\prime}\right\|_{\alpha} .
$$

Thus $\left\{\|\bullet, \bullet, \ldots, \bullet\|_{\alpha}: \alpha \in(0,1)\right\}$ is an $\alpha$-n-norm on $X$.

Let $0<\alpha_{1}<\alpha_{2}$. Then,

$$
\begin{aligned}
& \left\|f_{1}, f_{2}, \ldots, f_{n}\right\|_{\alpha_{1}}=\inf \left\{t: N\left(f_{1}, f_{2}, \ldots, f_{n}, t\right) \geq \alpha_{1}\right\}, \\
& \left\|f_{1}, f_{2}, \ldots, f_{n}\right\|_{\alpha_{2}}=\inf \left\{t: N\left(f_{1}, f_{2}, \ldots, f_{n}, t\right) \geq \alpha_{2}\right\} .
\end{aligned}
$$

As $\alpha_{1}<\alpha_{2}$,

$$
\left\{t: N\left(f_{1}, f_{2}, \ldots, f_{n}, t\right) \geq \alpha_{2}\right\} \subset\left\{t: N\left(f_{1}, f_{2}, \ldots, f_{n}, t\right) \geq \alpha_{1}\right\}
$$

implies that

$$
\inf \left\{t: N\left(f_{1}, f_{2}, \ldots, f_{n}, t\right) \geq \alpha_{2}\right\} \geq \inf \left\{t: N\left(f_{1}, f_{2}, \ldots, f_{n}, t\right) \geq \alpha_{1}\right\}
$$

which implies that

$$
\left\|f_{1}, f_{2}, \ldots, f_{n}\right\|_{\alpha_{2}} \geq\left\|f_{1}, f_{2}, \ldots, f_{n}\right\|_{\alpha_{1}} .
$$

Hence $\left\{\|\bullet, \bullet, \ldots, \bullet\|_{\alpha}: \alpha \in(0,1)\right\}$ is an ascending family of $\alpha$-n-norms on $x$ corresponding to the 2-fuzzy $n$-norm on $X$.

\section{On the Mazur-Ulam problem}

In this section, we give a new generalization of the Mazur-Ulam theorem when $X$ is a 2 -fuzzy $n$-normed linear space or $\mathfrak{I}(X)$ is a fuzzy $n$-normed linear space. Hereafter, we use the notion of fuzzy $n$-normed linear space on $\mathfrak{S}(X)$ instead of 2 -fuzzy $n$-normed linear space on $X$.

Definition 4.1 Let $\mathfrak{I}(X)$ and $\mathfrak{I}(X)$ be fuzzy $n$-normed linear spaces and $\Psi: \mathfrak{I}(X) \rightarrow \mathfrak{I}$ $(Y)$ a mapping. We call $\Psi$ an $n$-isometry if

$$
\left\|f_{1}-f_{0}, \ldots, f_{n}-f_{0}\right\|_{\alpha}=\left\|\Psi\left(f_{1}\right)-\Psi\left(f_{0}\right), \ldots, \Psi\left(f_{n}\right)-\Psi\left(f_{0}\right)\right\|_{\beta}
$$

for all $f_{0}, f_{1}, f_{2}, \ldots, f_{n} \in \mathfrak{S}(X)$ and $\alpha, \beta \in(0,1)$.

For a mapping $\Psi$, consider the following condition which is called the $n$-distance one preserving property $(n \mathrm{DOPP})$. 
( $n$ DOPP) Let $f_{0}, f_{1}, f_{2}, \ldots, f_{n} \in \Im(X)$ with $\left\|f_{1}-f_{0}, \ldots, f_{n}-f_{0}\right\|_{\alpha}=1$.

Then $\left\|\Psi\left(f_{1}\right)-\Psi\left(f_{0}\right), \ldots, \Psi\left(f_{n}\right)-\Psi\left(f_{0}\right)\right\|_{\beta}=1$.

Lemma 4.1 Let $f_{1}, f_{2}, \ldots . f_{n} \in \mathfrak{I}(X), \alpha \in(0,1)$ and $\hbar \in \mathbb{R}$. Then,

$$
\left\|f_{1}, \ldots, f_{i}, \ldots, f_{j}, \ldots, f_{n}\right\|_{\alpha}=\left\|f_{1}, \ldots, f_{i}, \ldots, f_{j}+\hbar f_{i}, \ldots, f_{n}\right\|_{\alpha}
$$

for all $1 \leq i \neq j \leq n$.

Proof. It is obviously true.

Lemma 4.2 For $f_{0}, f_{0}^{\prime} \in \Im(X)$, if $f_{0}$ and $f_{0}^{\prime}$ are linearly dependent with some direction, that is, $f_{0}^{\prime}=t f_{0}$ for some $t>0$, then

$$
\left\|f_{0}+f_{0}^{\prime}, f_{1}, \ldots, f_{n}\right\|_{\alpha}=\left\|f_{0}, f_{1}, \ldots, f_{n}\right\|_{\alpha}+\left\|f_{0}^{\prime}, f_{1}, \ldots, f_{n}\right\|_{\alpha}
$$

for all $f_{1}, f_{2}, \ldots, f_{n} \in \mathfrak{S}(X)$ and $\alpha \in(0,1)$.

Proof. Let $f_{0}^{\prime}=t f_{0}$ for some $t>0$. Then we have

$$
\begin{aligned}
\left\|f_{0}+f^{\prime}{ }_{0}, f_{1}, \ldots, f_{n}\right\|_{\alpha} & =\left\|f_{0}+t f_{0}, f_{1}, \ldots, f_{n}\right\|_{\alpha} \\
& =(1+t)\left\|f_{0}, f_{1}, \ldots, f_{n}\right\|_{\alpha} \\
& =\left\|f_{0}, f_{1}, \ldots, f_{n}\right\|_{\alpha}+t \mid\left\|f_{0}, f_{1}, \ldots, f_{n}\right\|_{\alpha} \\
& =\left\|f_{0}, f_{1}, \ldots, f_{n}\right\|_{\alpha}+\left\|f^{\prime}{ }_{0}, f_{1}, \ldots, f_{n}\right\|_{\alpha}
\end{aligned}
$$

for all $f_{1}, f_{2}, \ldots, f_{n} \in \mathfrak{S}(X)$ and $\alpha \in(0,1)$.

Definition 4.2 The elements $f_{0}, f_{1}, f_{2}, \ldots, f_{n}$ of $\mathfrak{S}(X)$ are said to be $n$-collinear if for every $i,\left\{f_{j}-f_{i}: 0 \leq j \neq i \leq n\right\}$ is linearly dependent.

Remark 4.1 The elements $f_{0}, f_{1}$, and $f_{2}$ are said to be 2-collinear if and only if $f_{2}-f_{0}$ $=r\left(f_{1}-f_{0}\right)$ for some real number $r$.

Now we define the concept of $n$-Lipschitz mapping.

Definition 4.3 We call $\Psi$ an $n$-Lipschitz mapping if there is a $\kappa \geq 0$ such that

$$
\left\|\Psi\left(f_{1}\right)-\Psi\left(f_{0}\right), \ldots, \Psi\left(f_{n}\right)-\Psi\left(f_{0}\right)\right\|_{\beta} \leq k|| f_{1}-f_{0}, \ldots, f_{n}-f_{0} \|_{\alpha}
$$

for all $f_{0}, f_{1}, f_{2}, \ldots, f_{n} \in \mathfrak{I}(X)$ and $\alpha, \beta \in(0,1)$. The smallest such $\kappa$ is called the $n$ Lipschitz constant.

Lemma 4.3 Assume that if $f_{0}, f_{1}$, and $f_{2}$ are 2 -collinear then $\Psi\left(f_{0}\right), \Psi\left(f_{1}\right)$ and $\Psi\left(f_{2}\right)$ are 2-collinear, and that $\Psi$ satisfies ( $n$ DOPP). Then $\Psi$ preserves the $n$-distance $k$ for each $k \in \mathbb{N}$.

Proof. Suppose that there exist $f_{0}, f_{1} \in \mathfrak{I}(X)$ with $f_{0} \neq f_{1}$ such that $\Psi\left(f_{0}\right)=\Psi\left(f_{1}\right)$. Since $\operatorname{dim} \mathfrak{I}(X) \geq n$, there are $f_{2}, \ldots, f_{n} \in \mathfrak{I}(X)$ such that $f_{1}-f_{0}, f_{2}-f_{0}, \ldots, f_{n}-f_{0}$ are linearly independent. Since $\left\|f_{1}-f_{0}, f_{2}-f_{0}, \ldots, f_{n}-f_{0}\right\|_{\alpha} \neq 0$, we can set

$$
z_{2}=f_{0}+\frac{f_{2}-f_{0}}{\left\|f_{1}-f_{0}, f_{2}-f_{0}, \ldots, f_{n}-f_{0}\right\|_{\alpha}} .
$$

Then we have

$$
\begin{aligned}
& \left\|f_{1}-f_{0}, z_{2}-f_{0}, f_{3}-f_{0}, \ldots, f_{n}-f_{0}\right\|_{\alpha} \\
& \quad=\left\|f_{1}-f_{0}, \frac{f_{2}-f_{0}}{\left\|f_{1}-f_{0}, f_{2}-f_{0}, \ldots, f_{n}-f_{0}\right\|_{\alpha}}, f_{3}-f_{0}, \ldots, f_{n}-f_{0}\right\|_{\alpha}=1 .
\end{aligned}
$$


Since $\Psi$ preserves the unit $n$-distance,

$$
\left\|\Psi\left(f_{1}\right)-\Psi\left(f_{0}\right), \Psi\left(z_{2}\right)-\Psi\left(f_{0}\right), \ldots, \Psi\left(f_{n}\right)-\Psi\left(f_{0}\right)\right\|_{\beta}=1 .
$$

But it follows from $\Psi\left(f_{0}\right)=\Psi\left(f_{1}\right)$ that

$$
\left\|\Psi\left(f_{1}\right)-\Psi\left(f_{0}\right), \Psi\left(z_{2}\right)-\Psi\left(f_{0}\right), \ldots, \Psi\left(f_{n}\right)-\Psi\left(f_{0}\right)\right\|_{\beta}=0,
$$

which is a contradiction. Hence, $\Psi$ is injective.

Let $f_{0}, f_{1}, f_{2}, \ldots, f_{n}$ be elements of $\Im(X), k \in \mathbb{N}$ and

$$
\left\|f_{1}-f_{0}, f_{2}-f_{0}, \ldots, f_{n}-f_{0}\right\|_{\alpha}=k .
$$

We put

$$
g_{i}=f_{0}+\frac{i}{k}\left(f_{1}-f_{0}\right), \quad i=0,1, \ldots, k .
$$

Then

$$
\begin{aligned}
& \left\|g_{i+1}-g_{i}, f_{2}-f_{0}, \ldots, f_{n}-f_{0}\right\|_{\alpha} \\
& \quad=\left\|f_{0}+\frac{i+1}{k}\left(f_{1}-f_{0}\right)-\left(f_{0}+\frac{i}{k}\left(f_{1}-f_{0}\right)\right), f_{2}-f_{0}, \ldots, f_{n}-f_{0}\right\|_{\alpha} \\
& \quad=\left\|\frac{1}{k}\left(f_{1}-f_{0}\right), f_{2}-f_{0}, \ldots, f_{n}-f_{0}\right\|_{\alpha} \\
& \quad=\frac{i}{k}\left\|f_{1}-f_{0}, f_{2}-f_{0}, \ldots, f_{n}-f_{0}\right\|_{\alpha}=\frac{k}{k}=1
\end{aligned}
$$

for all $i=0,1, \ldots, k-1$. Since $\Psi$ satisfies (nDOPP),

$$
\left\|\Psi\left(g_{i+1}\right)-\Psi\left(g_{i}\right), \Psi\left(f_{2}\right)-\Psi\left(f_{0}\right) \ldots, \Psi\left(f_{n}\right)-\Psi\left(f_{0}\right)\right\|_{\beta}=1
$$

for all $i=0,1, \ldots, k-1$. Since $g_{0}, g_{1}$, and $g_{2}$ are 2-collinear, $\Psi\left(g_{0}\right), \Psi\left(g_{1}\right)$ and $\Psi\left(g_{2}\right)$ are also 2-collinear. Thus there is a real number $r_{0}$ such that $\Psi\left(g_{2}\right)-\Psi\left(g_{1}\right)=r_{0}\left(\Psi\left(g_{1}\right)\right.$ $\left.\Psi\left(g_{0}\right)\right)$. It follows from (4.1) that

$$
\begin{aligned}
\left\|\Psi\left(g_{1}\right)-\Psi\left(g_{0}\right), \Psi\left(f_{2}\right)-\Psi\left(f_{0}\right), \ldots, \Psi\left(f_{n}\right)-\Psi\left(f_{0}\right)\right\|_{\beta} \\
\quad=\left\|\Psi\left(g_{2}\right)-\Psi\left(g_{1}\right), \Psi\left(f_{2}\right)-\Psi\left(f_{0}\right), \ldots, \Psi\left(f_{n}\right)-\Psi\left(f_{0}\right)\right\|_{\beta} \\
\quad=\left\|r_{0}\left(\Psi\left(g_{1}\right)-\Psi\left(g_{2}\right)\right), \Psi\left(f_{2}\right)-\Psi\left(f_{0}\right), \ldots, \Psi\left(f_{n}\right)-\Psi\left(f_{0}\right)\right\|_{\beta} \\
\quad=\left|r_{0}\right|\left\|\left(\Psi\left(g_{1}\right)-\Psi\left(g_{0}\right)\right), \Psi\left(f_{2}\right)-\Psi\left(f_{0}\right), \ldots, \Psi\left(f_{n}\right)-\Psi\left(f_{0}\right)\right\|_{\beta} .
\end{aligned}
$$

Thus, we have $r_{0}=1$ or -1 . If $r_{0}=-1, \Psi\left(g_{2}\right)-\Psi\left(g_{1}\right)=-\Psi\left(g_{1}\right)+\Psi\left(g_{0}\right)$, that is, $\Psi\left(g_{2}\right)=$ $\Psi\left(g_{0}\right)$. Since $\Psi$ is injective, $g_{2}=g_{0}$, which is a contradiction. Thus $r_{0}=1$. Then we have $\Psi\left(g_{2}\right)-\Psi\left(g_{1}\right)=\Psi\left(g_{1}\right)-\Psi\left(g_{0}\right)$. Similarly, one can obtain that $\Psi\left(g_{i+1}\right)-\Psi\left(g_{i}\right)=\Psi\left(g_{i}\right)-$ $\Psi\left(g_{i-1}\right)$ for all $i=0,1, \ldots, k-1$. Thus $\Psi\left(g_{i+1}\right)-\Psi\left(g_{i}\right)=\Psi\left(g_{1}\right)-\Psi\left(g_{0}\right)$ for all $i=0,1, \ldots, k$ - 1. Hence

$$
\begin{aligned}
& \Psi\left(f_{1}\right)-\Psi\left(f_{0}\right)=\Psi\left(g_{k}\right)-\Psi\left(g_{0}\right) \\
& \quad=\Psi\left(g_{k}\right)-\Psi\left(g_{k-1}\right)+\Psi\left(g_{k-1}\right)-\Psi\left(g_{k-2}\right)+\cdots+\Psi\left(g_{1}\right)-\Psi\left(g_{0}\right) \\
& \quad=k\left(\Psi\left(g_{1}\right)-\Psi\left(g_{0}\right)\right) .
\end{aligned}
$$


Hence

$$
\begin{aligned}
& \left\|\Psi\left(f_{1}\right)-\Psi\left(f_{0}\right), \Psi\left(f_{2}\right)-\Psi\left(f_{0}\right), \ldots, \Psi\left(f_{n}\right)-\Psi\left(f_{0}\right)\right\|_{\beta} \\
& \quad=\left\|k\left(\Psi\left(g_{1}\right)-\Psi\left(g_{0}\right)\right), \Psi\left(f_{2}\right)-\Psi\left(f_{0}\right), \ldots, \Psi\left(f_{n}\right)-\Psi\left(f_{0}\right)\right\|_{\beta} \\
& \quad=\left\|k \Psi\left(g_{1}\right)-\Psi\left(g_{0}\right), \Psi\left(f_{2}\right)-\Psi\left(f_{0}\right), \ldots, \Psi\left(f_{n}\right)-\Psi\left(f_{0}\right)\right\|_{\beta}=k .
\end{aligned}
$$

This completes the proof.

Lemma 4.4 Let $h, f_{0}, f_{1}, \ldots, f_{n}$ be elements of $\mathfrak{I}(X)$ and let $h, f_{0}, f_{1}$ be 2 -collinear. Then

$$
\left\|f_{1}-h, f_{2}-h, \ldots, f_{n}-h\right\|_{\alpha}=\left\|f_{1}-h, f_{2}-f_{0}, \ldots, f_{n}-f_{0}\right\|_{\alpha} .
$$

Proof. Since $h, f_{0}, f_{1}$ are 2-collinear, there exists a real number $r$ such that $f_{1}-h=r\left(f_{0}\right.$ - h). It follows from Lemma 4.1 that

$$
\begin{aligned}
\left\|f_{1}-h, f_{2}-f_{0}, \ldots, f_{n}-f_{0}\right\|_{\alpha} & =\left\|r\left(f_{0}-h\right), f_{2}-f_{0}, \ldots, f_{n}-f_{0}\right\|_{\alpha} \\
& =|r|\left\|f_{0}-h, f_{2}-f_{0}, \ldots, f_{n}-f_{0}\right\|_{\alpha} \\
& =|r|\left\|f_{0}-h, f_{2}-h, \ldots, f_{n}-h\right\| \|_{\alpha} \\
& =\left\|r\left(f_{0}-h\right), f_{2}-h, \ldots, f_{n}-h \mid\right\|_{\alpha} \\
& =\left\|f_{1}-h, f_{2}-h, \ldots, f_{n}-h\right\|_{\alpha} .
\end{aligned}
$$

This completes the proof.

Theorem 4.1 Let $\Psi$ be an $n$-Lipschitz mapping with the $n$-Lipschitz constant $\kappa \leq 1$. Assume that if $f_{0}, f_{1}, \ldots, f_{n}$ are $m$-collinear then $\Psi\left(f_{0}\right), \Psi\left(f_{1}\right), \ldots, \Psi\left(f_{m}\right)$ are $m$-collinear, $m$ $=2, n$, and that $\Psi$ satisfies ( $n \mathrm{DOPP})$, then $\Psi$ is an $n$-isometry.

Proof. It follows from Lemma 4.3 that $\Psi$ preserves $n$-distance $k$ for all $k \in \mathbb{N}$. For $f_{0}$, $f_{1}, \ldots, f_{n} \in X$, there are two cases depending upon whether $\left\|f_{1}-f_{0}, \ldots, f_{n}-f_{0}\right\|_{\alpha}=0$ or not. In the case $\left\|f_{1}-f_{0}, \ldots, f_{n}-f_{0}\right\|_{\alpha}=0, f_{1}-f_{0}, \ldots, f_{n}-f_{0}$ are linearly dependent, that is, $n$-collinear. Thus $f_{1}-f_{0}, \ldots, f_{n}-f_{0}$ are linearly dependent. Thus $\| \Psi\left(f_{1}\right)-\Psi\left(f_{0}\right), \ldots, \Psi\left(f_{n}\right)-$ $\Psi\left(f_{0}\right) \|_{\beta}=0$.

In the case $\left\|f_{1}-f_{0}, \ldots, f_{n}-f_{0}\right\|_{\alpha}>0$, there exists an $n_{0} \in \mathbb{N}$ such that

$$
n_{0}>\left\|f_{1}-f_{0}, \ldots, f_{n}-f_{0}\right\|_{\alpha}
$$

Assume that

$$
\left\|\Psi\left(f_{1}\right)-\Psi\left(f_{0}\right), \ldots, \Psi\left(f_{n}\right)-\Psi\left(f_{0}\right)\right\|_{\beta}<\left\|f_{1}-f_{0}, \ldots, f_{n}-f_{0}\right\|_{\alpha} .
$$

We can set

$$
h=f_{0}+\frac{n_{0}}{\left\|f_{1}-f_{0}, \ldots, f_{n}-f_{0}\right\|_{\alpha}}\left(f_{1}-f_{0}\right) .
$$

Then we get

$$
\begin{aligned}
\| h & -f_{0}, \ldots, f_{n}-f_{0} \|_{\alpha} \\
& =\left\|f_{0}+\frac{n_{0}}{\left\|f_{1}-f_{0}, \ldots, f_{n}-f_{0}\right\|_{\alpha}}\left(f_{1}-f_{0}\right)-f_{0}, \ldots, f_{n}-f_{0}\right\|_{\alpha} \\
& =\frac{n_{0}}{\left\|f_{1}-f_{0}, \ldots, f_{n}-f_{0}\right\|_{\alpha}}\left\|f_{1}-f_{0}, \ldots, f_{n}-f_{0}\right\|_{\alpha}=n_{0} .
\end{aligned}
$$

It follows from Lemma 4.3 that

$$
\left\|\Psi(h)-\Psi\left(f_{0}\right), \ldots, \Psi\left(f_{n}\right)-\Psi\left(f_{0}\right)\right\|_{\beta}=n_{0} .
$$


By the definition of $h$,

$$
h-f_{1}=\left(\frac{n_{0}}{\left\|f_{1}-f_{0}, \ldots, f_{n}-f_{0}\right\|_{\alpha}}-1\right)\left(f_{1}-f_{0}\right) .
$$

Since

$$
\frac{n_{0}}{\left\|f_{1}-f_{0}, \ldots, f_{n}-f_{0}\right\|_{\alpha}}>1
$$

$h-f_{1}$ and $f_{1}-f_{0}$ have the same direction. It follows from Lemma 4.2 that

$$
\begin{aligned}
& \left\|h-f_{0}, f_{2}-f_{0}, \ldots, f_{n}-f_{0}\right\|_{\alpha} \\
& \quad=\left\|h-f_{1}, f_{2}-f_{0}, \ldots, f_{n}-f_{0}\right\|_{\alpha}+\left\|f_{1}-f_{0}, f_{2}-f_{0}, \ldots, f_{n}-f_{0}\right\|_{\alpha} .
\end{aligned}
$$

Since $\Psi(h), \Psi\left(f_{1}\right), \Psi\left(f_{2}\right)$ are 2-collinear, we have

$$
\begin{aligned}
& \left\|\Psi(h)-\Psi\left(f_{1}\right), \Psi\left(f_{2}\right)-\Psi\left(f_{0}\right), \ldots, \Psi\left(f_{n}\right)-\Psi\left(f_{0}\right)\right\|_{\beta} \\
& \quad=\left\|\Psi\left(f_{1}\right)-\Psi(h), \Psi\left(f_{2}\right)-\Psi(h), \ldots, \Psi\left(f_{n}\right)-\Psi(h)\right\|_{\beta} \\
& \quad \leq\left\|f_{1}-h, f_{2}-h, \ldots, f_{n}-h\right\|_{\alpha} \\
& \quad=\left\|f_{1}-h, f_{2}-f_{0}, \ldots, f_{n}-f_{0}\right\|_{\alpha} \\
& \quad=n_{0}-\left\|f_{1}-f_{0}, f_{2}-f_{0}, \ldots, f_{n}-f_{0}\right\|_{\alpha}
\end{aligned}
$$

by Lemma 4.4. By the assumption,

$$
\begin{aligned}
n_{0}= & \left\|\Psi(h)-\Psi\left(f_{0}\right), \Psi\left(f_{2}\right)-\Psi\left(f_{0}\right), \ldots, \Psi\left(f_{n}\right)-\Psi\left(f_{0}\right)\right\|_{\beta} \\
\leq & \left\|\Psi(h)-\Psi\left(f_{1}\right), \Psi\left(f_{2}\right)-\Psi\left(f_{0}\right), \ldots, \Psi\left(f_{n}\right)-\Psi\left(f_{0}\right)\right\|_{\beta} \\
& +\left\|\Psi\left(f_{1}\right)-\Psi\left(f_{0}\right), \Psi\left(f_{2}\right)-\Psi\left(f_{0}\right), \ldots, \Psi\left(f_{n}\right)-\Psi\left(f_{0}\right)\right\|_{\beta} \\
< & n_{0}-\left\|f_{1}-f_{0}, f_{2}-f_{0}, \ldots, f_{n}-f_{0}\right\|_{\alpha} \\
& +\left\|f_{1}-f_{0}, f_{2}-f_{0}, \ldots, f_{n}-f_{0}\right\| \alpha \\
= & n_{0},
\end{aligned}
$$

which is a contradiction. Hence $\Psi$ is an $n$-isometry.

Lemma 4.5 Let $g_{0}, g_{1}$ be elements of $\Im(X)$. Then $v=\frac{g_{0}+g_{1}}{2}$ is the unique element of $\mathfrak{I}(X)$ satisfying

$$
\begin{aligned}
& \frac{1}{2}\left\|g_{0}-g_{n}, g_{1}-g_{n}, g_{2}-g_{n}, \ldots, g_{n-1}-g_{n}\right\|_{\alpha} \\
& =\left\|g_{1}-v, g_{1}-g_{n}, g_{2}-g_{n}, \ldots, g_{n-1}-g_{n}\right\|_{\alpha} \\
& =\left\|g_{0}-g_{n}, g_{0}-v, g_{2}-g_{n}, \ldots, g_{n-1}-g_{n}\right\|_{\alpha}
\end{aligned}
$$

for some $g_{2}, \ldots, g_{n} \in \mathfrak{I}(X)$ with $\left\|g_{0}-g_{n}, g_{1}-g_{n}, g_{2}-g_{n}, \ldots, g_{n-1}-g_{n}\right\|_{\alpha} \neq 0$ and $v, g_{0}, g_{1} 2$ collinear.

Proof. Let $\left\|g_{0}-g_{n}, g_{1}-g_{n}, g_{2}-g_{n}, \ldots, g_{n-1}-g_{n}\right\|_{\alpha} \neq 0$ and $\left\|f_{0}-f_{n}, f_{1}-f_{n}, f_{2}-f_{n}, \ldots, f_{n-1}-f_{n}\right\|_{\alpha} \neq 0$. .

Then $v, g_{0}, g_{1}$ are 2-collinear. It follows from Lemma 4.1 and $g_{n}-g_{0}=g_{1}-g_{0}-\left(g_{1}-\right.$ $\left.g_{n}\right)$ that 


$$
\begin{aligned}
\left\|g_{1}-v, g_{1}-g_{n}, g_{2}-g_{n}, \ldots g_{n-1}-g_{n}\right\|_{\alpha} & \\
= & \left\|g_{1} \frac{g_{0}+g_{1}}{2}, g_{1}-g_{n}, g_{2}-g_{n}, \ldots, g_{n-1}-g_{n}\right\|_{\alpha} \\
= & \frac{1}{2}\left\|g_{1}-g_{0}, g_{1}-g_{n}, g_{2}-g_{n}, \ldots, g_{n-1}-g_{n}\right\|_{\alpha} \\
= & \frac{1}{2}\left\|g_{0}-g_{n}, g_{1}-g_{n}, g_{2}-g_{n}, \ldots, g_{n-1}-g_{n}\right\| \alpha
\end{aligned}
$$

and similarly

$$
\left\|g_{0}-g_{n}, g_{0}-v, g_{2}-g_{n}, \ldots, g_{n-1}-g_{n}\right\|_{\alpha}=\frac{1}{2}\left\|g_{0}-g_{n}, g_{1}-g_{n}, g_{2}-g_{n}, \ldots, g_{n-1}-g_{n}\right\|_{\alpha} .
$$

Now we prove the uniqueness.

Let $u$ be an element of $\mathfrak{I}(X)$ satisfying the above properties. Since $u, g_{0}, g_{1}$ are 2-collinear, there exists a real number $t$ such that $u=t g_{0}+(1-t) g_{1}$. It follows from Lemma 4.1 that

$$
\begin{aligned}
\frac{1}{2} & \left\|g_{0}-g_{n}, g_{1}-g_{n}, g_{2}-g_{n}, \ldots, g_{n-1}-g_{n}\right\|_{\alpha} \\
& =\left\|g_{1}-u, g_{1}-g_{n}, g_{2}-g_{n}, \ldots, g_{n-1}-g_{n}\right\|_{\alpha} \\
& =\left\|g_{1}-\left(t g_{0}+(1-t) g_{1}\right), g_{1}-g_{n}, g_{2}-g_{n}, \ldots, g_{n-1}-g_{n}\right\|_{\alpha} \\
& =|t|\left\|g_{1}-g_{0}, g_{1}-g_{n}, g_{2}-g_{n}, \ldots, g_{n-1}-g_{n}\right\|_{\alpha} \\
& =|t|\left\|g_{0}-g_{n}, g_{1}-g_{n}, g_{2}-g_{n}, \ldots, g_{n-1}-g_{n}\right\|_{\alpha}
\end{aligned}
$$

and

$$
\begin{aligned}
\frac{1}{2} & \left\|g_{0}-g_{n}, g_{1}-g_{n}, g_{2}-g_{n}, \ldots, g_{n-1}-g_{n}\right\|_{\alpha} \\
& =|| g_{0}-g_{n}, g_{0}-u, g_{2}-g_{n}, \ldots, g_{n-1}-g_{n} \|_{\alpha} \\
& =|| g_{0}-g_{n}, g_{0}-\left(t g_{0}+(1-t) g_{1}\right), g_{2}-g_{n}, \ldots, g_{n-1}-g_{n} \|_{\alpha} \\
& =|1-t|\left\|g_{0}-g_{n}, g_{0}-g_{1}, g_{2}-g_{n}, \ldots, g_{n-1}-g_{n}\right\|_{\alpha} \\
& =|1-t|\left\|g_{0}-g_{n}, g_{1}-g_{n}, g_{2}-g_{n}, \ldots, g_{n-1}-g_{n}\right\|_{\alpha} .
\end{aligned}
$$

Since $\left\|g_{0}-g_{n}, g_{1}-g_{n}, g_{2}-g_{n}, \ldots, g_{n-1}-g_{n}\right\|_{\alpha} \neq 0$, we have $\frac{1}{2}=|1-t|=|t|$. Therefore, we get $t=\frac{1}{2}$ and hence $v=u$.

Lemma 4.6 If $\Psi$ is an $n$-isometry and $f_{0}, f_{1}, f_{2}$ are 2-collinear then $\Psi\left(f_{0}\right), \Psi\left(f_{1}\right), \Psi\left(f_{2}\right)$ are 2-collinear.

Proof. Since $\operatorname{dim} \mathfrak{I}(X) \geq n$, for any $f_{0} \in \mathfrak{I}(X)$, there exist $g_{1}, \ldots, g_{n} \in \mathfrak{I}(X)$ such that $g_{1}$ $f_{0}, \ldots, g_{n}-f_{0}$ are linearly independent. Then

$$
\left\|g_{1}-f_{0}, \ldots, g_{n}-f_{0}\right\|_{\alpha}=\left\|\Psi\left(g_{1}\right)-\Psi\left(f_{0}\right), \ldots, \Psi\left(g_{n}\right)-\Psi\left(f_{0}\right)\right\|_{\beta} \neq 0
$$

and hence, the set $A=\left\{\Psi(f)-\Psi\left(f_{0}\right): f \in \mathfrak{I}(X)\right\}$ contains $n$ linearly independent vectors.

Assume that $f_{0}, f_{1}, f_{2}$ are 2 -collinear. Then, for any $f_{3}, \ldots, f_{n} \in \mathfrak{I}(X)$,

$$
\left\|f_{1}-f_{0}, \ldots, f_{n}-f_{0}\right\|_{\alpha}=\left\|\Psi\left(f_{1}\right)-\Psi\left(f_{0}\right), \ldots, \Psi\left(f_{n}\right)-\Psi\left(f_{0}\right)\right\|_{\beta}=0,
$$

i.e. $\Psi\left(f_{1}\right)-\Psi\left(f_{0}\right), \ldots, \Psi\left(f_{n}\right)-\Psi\left(f_{0}\right)$ are linearly dependent. 
If there exist $f_{3}, \ldots, f_{n-1}$ such that $\Psi\left(f_{1}\right)-\Psi\left(f_{0}\right), \ldots, \Psi\left(f_{n-1}\right)-\Psi\left(f_{0}\right)$ are linearly independent, then

$$
A=\left\{\Psi\left(f_{n}\right)-\Psi\left(f_{0}\right): f_{n} \in \Im(X)\right\} \subset \operatorname{span}\left\{\Psi\left(f_{1}\right)-\Psi\left(f_{0}\right), \ldots, \Psi\left(f_{n-1}\right)-\Psi\left(f_{0}\right)\right\},
$$

which contradicts the fact that $A$ contains $n$ linearly independent vectors.

Then, for any $f_{3}, \ldots, f_{n-1}, \Psi\left(f_{1}\right)-\Psi\left(f_{0}\right), \ldots, \Psi\left(f_{n-1}\right)-\Psi\left(f_{0}\right)$ are linearly dependent.

If there exist $f_{3}, \ldots, f_{n-2}$ such that $\Psi\left(f_{1}\right)-\Psi\left(f_{0}\right), \ldots, \Psi\left(f_{n-2}\right)-\Psi\left(f_{0}\right)$ are linearly independent, then

$$
A=\left\{\Psi\left(f_{n-1}\right)-\Psi\left(f_{0}\right): f_{n-1} \in \Im(X)\right\} \subset \operatorname{span}\left\{\Psi\left(f_{1}\right)-\Psi\left(f_{0}\right), \ldots, \Psi\left(f_{n-2}\right)-\Psi\left(f_{0}\right)\right\},
$$

which contradicts the fact that $A$ contains $n$ linearly independent vectors.

And so on, $\Psi\left(f_{1}\right)-\Psi\left(f_{0}\right), \Psi\left(f_{2}\right)-\Psi\left(f_{0}\right)$ are linearly dependent. Thus $\Psi\left(f_{0}\right), \Psi\left(f_{1}\right)$, and $\Psi\left(f_{2}\right)$ are 2-collinear.

Theorem 4.2 Every $n$-isometry mapping is affine.

Proof. Let $\Psi$ be an $n$-isometry and $\Phi(f)=\Psi(f)-\Psi(0)$. Then $\Phi$ is an $n$-isometry and $\Phi$ $(0)=0$. Thus we may assume that $\Psi(0)=0$. Hence it suffices to show that $\Psi$ is linear.

Let $f_{0}, f_{1} \in \mathfrak{I}(X)$ with $f_{0} \neq f_{1}$. Since $\operatorname{dim} \mathfrak{I}(X) \geq n$, there exist $f_{2}, \ldots, f_{n} \in \mathfrak{I}(X)$ such that

$$
\left\|f_{0}-f_{n}, f_{1}-f_{n}, f_{2}-f_{n}, \ldots, f_{n-1}-f_{n}\right\|_{\alpha} \neq 0 .
$$

Since $\Psi$ is an $n$-isometry, we have

$$
\left\|\Psi\left(f_{0}\right)-\Psi\left(f_{n}\right), \Psi\left(f_{1}\right)-\Psi\left(f_{n}\right), \Psi\left(f_{2}\right)-\Psi\left(f_{n}\right), \ldots, \Psi\left(f_{n-1}\right)-\Psi\left(f_{n}\right)\right\|_{\beta} \neq 0 .
$$

It follows from Lemma 4.1 that

$$
\begin{aligned}
& \left\|\Psi\left(f_{0}\right)-\Psi\left(f_{n}\right), \Psi\left(f_{0}\right)-\Psi\left(\frac{f_{0}+f_{1}}{2}\right), \Psi\left(f_{2}\right)-\Psi\left(f_{n}\right), \ldots, \Psi\left(f_{n-1}\right)-\Psi\left(f_{n}\right)\right\|_{\beta} \\
& =\left\|\Psi\left(f_{n}\right)-\Psi\left(f_{0}\right), \Psi\left(\frac{f_{0}+f_{1}}{2}\right)-\Psi\left(f_{0}\right), \Psi\left(f_{2}\right)-\Psi\left(f_{0}\right), \ldots, \Psi\left(f_{n-1}\right)-\Psi\left(f_{0}\right)\right\|_{\beta} \\
& =\left\|f_{n}-f_{0}, \frac{f_{0}+f_{1}}{2}-f_{0}, f_{2}-f_{0}, \ldots, f_{n-1}-f_{0}\right\|_{\alpha} \\
& =\frac{1}{2}\left\|f_{n}-f_{0}, f_{1}-f_{0}, f_{2}-f_{0}, \ldots, f_{n-1}-f_{0}\right\|_{\alpha} \\
& =\frac{1}{2}\left\|\Psi\left(f_{n}\right)-\Psi\left(f_{0}\right), \Psi\left(f_{1}\right)-\Psi\left(f_{0}\right), \Psi\left(f_{2}\right)-\Psi\left(f_{0}\right), \ldots, \Psi\left(f_{n-1}\right)-\Psi\left(f_{0}\right)\right\|_{\beta} \\
& =\frac{1}{2}\left\|\Psi\left(f_{0}\right)-\Psi\left(f_{n}\right), \Psi\left(f_{1}\right)-\Psi\left(f_{n}\right), \Psi\left(f_{2}\right)-\Psi\left(f_{n}\right), \ldots, \Psi\left(f_{n-1}\right)-\Psi\left(f_{n}\right)\right\|_{\beta} .
\end{aligned}
$$

And we get

$$
\begin{aligned}
& \left\|\Psi\left(f_{1}\right)-\Psi\left(\frac{f_{0}+f_{1}}{2}\right), \Psi\left(f_{1}\right)-\Psi\left(f_{n}\right), \Psi\left(f_{2}\right)-\Psi\left(f_{n}\right), \ldots, \Psi\left(f_{n-1}\right)-\Psi\left(f_{n}\right)\right\|_{\beta} \\
& =\left\|\Psi\left(\frac{f_{0}+f_{1}}{2}\right)-\Psi\left(f_{1}\right), \Psi\left(f_{n}\right)-\Psi\left(f_{1}\right), \Psi\left(f_{2}\right)-\Psi\left(f_{1}\right), \ldots, \Psi\left(f_{n-1}\right)-\Psi\left(f_{1}\right)\right\|_{\beta} \\
& =\left\|\frac{f_{0}+f_{1}}{2}-f_{1}, f_{n}-f_{1}, f_{2}-f_{1}, \ldots, f_{n-1}-f_{1}\right\|_{\alpha} \\
& =\frac{1}{2}\left\|f_{0}-f_{1}, f_{n}-f_{1}, f_{2}-f_{1}, \ldots, f_{n-1}-f_{1}\right\|_{\alpha} \\
& =\frac{1}{2}\left\|\Psi\left(f_{0}\right)-\Psi\left(f_{1}\right), \Psi\left(f_{n}\right)-\Psi\left(f_{1}\right), \Psi\left(f_{2}\right)-\Psi\left(f_{1}\right), \ldots, \Psi\left(f_{n-1}\right)-\Psi\left(f_{1}\right)\right\|_{\beta} \\
& =\frac{1}{2}\left\|\Psi\left(f_{0}\right)-\Psi\left(f_{n}\right), \Psi\left(f_{1}\right)-\Psi\left(f_{n}\right), \Psi\left(f_{2}\right)-\Psi\left(f_{n}\right), \ldots, \Psi\left(f_{n-1}\right)-\Psi\left(f_{n}\right)\right\|_{\beta} .
\end{aligned}
$$


By Lemma 4.6, we obtain that $\Psi\left(\frac{f_{0}+f_{1}}{2}\right), \Psi\left(f_{0}\right)$, and $\Psi\left(f_{1}\right)$ are 2-collinear. By Lemma 4.5, we get $\Psi\left(\frac{f_{0}+f_{1}}{2}\right)=\frac{\Psi\left(f_{0}\right)+\Psi\left(f_{1}\right)}{2}$ for all $f, g \in \mathfrak{I}(X)$ and $\alpha, \beta \in(0,1)$. Since $\Psi(0)=0$, we can easily show that $\Psi$ is additive. It follows that $\Psi$ is $\mathbb{Q}$ - linear-linear.

Let $r \in \mathbb{R}^{+}$with $r \neq 1$ and $f \in \mathfrak{I}(X)$. By Lemma 4.6, $\Psi(0), \Psi(f)$ and $\Psi(r f)$ are also 2collinear. It follows from $\Psi(0)=0$ that there exists a real number $k$ such that $\Psi(r f)=$ $k \Psi(f)$. Since $\operatorname{dim} \mathfrak{I}(X) \geq n$, there exist $f_{1}, \ldots, f_{n-1} \in \mathfrak{I}(X)$ such that $\left\|f, f_{1}, f_{2}, \ldots, f_{n-1}\right\|_{\alpha} \neq 0$. Since $\Psi(0)=0$, for every $f_{0}, f_{1}, f_{2}, \ldots, f_{n-1} \in \mathfrak{S}(X)$,

$$
\begin{aligned}
& \left\|f_{0}, f_{1}, f_{2}, \ldots, f_{n-1}\right\|_{\alpha} \\
& \quad=\left\|f_{0}-0, f_{1}-0, f_{2}-0, \ldots, f_{n-1}-0\right\|_{\alpha} \\
& \quad=\left\|\Psi\left(f_{0}\right)-\Psi(0), \Psi\left(f_{1}\right)-\Psi(0), \Psi\left(f_{2}\right)-\Psi(0), \ldots, \Psi\left(f_{n-1}\right)-\Psi(0)\right\|_{\beta} \\
& \quad=\left\|\Psi\left(f_{0}\right), \Psi\left(f_{1}\right), \Psi\left(f_{2}\right), \ldots, \Psi\left(f_{n-1}\right)\right\|_{\beta} .
\end{aligned}
$$

Thus we have

$$
\begin{aligned}
r\left\|f, f_{1}, f_{2}, \ldots, f_{n-1}\right\|_{\alpha} & =\left\|r f, f_{1}, f_{2}, \ldots, f_{n-1}\right\|_{\alpha} \\
& =\left\|\Psi(r f), \Psi\left(f_{1}\right), \Psi\left(f_{2}\right), \ldots, \Psi\left(f_{n-1}\right)\right\|_{\beta} \\
& =\left\|k \Psi(f), \Psi\left(f_{1}\right), \Psi\left(f_{2}\right), \ldots, \Psi\left(f_{n-1}\right)\right\|_{\beta} \\
& =|k|\left\|\Psi(f), \Psi\left(f_{1}\right), \Psi\left(f_{2}\right), \ldots, \Psi\left(f_{n-1}\right)\right\|_{\beta} \\
& =|k|\left\|f, f_{1}, f_{2}, \ldots, f_{n-1}\right\|_{\alpha} .
\end{aligned}
$$

Since $\left\|f, f_{1}, f_{2}, \ldots, f_{n-1}\right\|_{\alpha} \neq 0,|k|=r$. Then $\Psi(r f)=r \Psi(f)$ or $\Psi(r f)=-r \Psi(f)$. First of all, assume that $k=-r$, that is, $\Psi(r f)=-r \Psi(f)$. Then there exist positive rational numbers $q_{1}, q_{2}$ such that $o<q_{1}<r<q_{2}$. Since $\operatorname{dim} \mathfrak{I}(X) \geq n$, there exist $h_{1}, \ldots, h_{n-1} \in \mathfrak{I}(X)$ such that

$$
\left\|r f-q_{2} f, h_{1}-q_{2} f, h_{2}-q_{2} f, \ldots, h_{n-1}-q_{2} f\right\|_{\alpha} \neq 0 .
$$

Then we have

$$
\begin{aligned}
& \left(q_{2}+r\right)\left\|\Psi(f), \Psi\left(h_{1}\right)-\Psi\left(q_{2} f\right), \Psi\left(h_{2}\right)-\Psi\left(q_{2} f\right), \ldots, \Psi\left(h_{n-1}\right)-\Psi\left(q_{2} f\right)\right\|_{\beta} \\
& \quad=\left\|\left(q_{2}+r\right) \Psi(f), \Psi\left(h_{1}\right)-\Psi\left(q_{2} f\right), \Psi\left(h_{2}\right)-\Psi\left(q_{2} f\right), \ldots, \Psi\left(h_{n-1}\right)-\Psi\left(q_{2} f\right)\right\|_{\beta} \\
& \quad=\left\|q_{2} \Psi(f)-(-r \Psi(f)), \Psi\left(h_{1}\right)-\Psi\left(q_{2} f\right), \Psi\left(h_{2}\right)-\Psi\left(q_{2} f\right), \ldots, \Psi\left(h_{n-1}\right)-\Psi\left(q_{2} f\right)\right\|_{\beta} \\
& \quad=\left\|\Psi(r f)-\Psi\left(q_{2} f\right), \Psi\left(h_{1}\right)-\Psi\left(q_{2} f\right), \Psi\left(h_{2}\right)-\Psi\left(q_{2} f\right), \ldots, \Psi\left(h_{n-1}\right)-\Psi\left(q_{2} f\right)\right\|_{\beta} \\
& \quad=\left\|r f-q_{2} f, h_{1}-q_{2} f, h_{2}-q_{2} f, \ldots, h_{n-1}-q_{2} f\right\|_{\alpha} \\
& \quad=\left(q_{2}-r\right)\left\|f, h_{1}-q_{2} f, h_{2}-q_{2} f, \ldots, h_{n-1}-q_{2} f\right\|_{\alpha} \\
& \quad \leq\left(q_{2}-q_{1}\right)\left\|f, h_{1}-q_{2} f, h_{2}-q_{2} f, \ldots, h_{n-1}-q_{2} f\right\|_{\alpha} \\
& \quad=\left\|q_{1} f-q_{2} f, h_{1}-q_{2} f, h_{2}-q_{2} f, \ldots, h_{n-1}-q_{2} f\right\|_{\alpha} \\
& \quad=\left\|\Psi\left(q_{1} f\right)-\Psi\left(q_{2} f\right), \Psi\left(h_{1}\right)-\Psi\left(q_{2} f\right), \Psi\left(h_{2}\right)-\Psi\left(q_{2} f\right), \ldots, \Psi\left(h_{n-1}\right)-\Psi\left(q_{2} f\right)\right\|_{\beta} .
\end{aligned}
$$

And also we have

$$
\begin{aligned}
& \left\|r f-q_{2} f, h_{1}-q_{2} f, h_{2}-q_{2} f, \ldots, h_{n-1}-q_{2} f\right\|_{\alpha} \\
& \quad=\left\|\Psi(r f)-\Psi\left(q_{2} f\right), \Psi\left(h_{1}\right)-\Psi\left(q_{2} f\right), \Psi\left(h_{2}\right)-\Psi\left(q_{2} f\right), \ldots, \Psi\left(h_{n-1}\right)-\Psi\left(q_{2} f\right)\right\|_{\beta} \\
& \quad=\left\|-r \Psi(f)-q_{2} \Psi(f), \Psi\left(h_{1}\right)-\Psi\left(q_{2} f\right), \Psi\left(h_{2}\right)-\Psi\left(q_{2} f\right), \ldots, \Psi\left(h_{n-1}\right)-\Psi\left(q_{2} f\right)\right\|_{\beta} \\
& \quad=\left(r+q_{2}\right)\left\|\Psi(f),, \Psi\left(h_{1}\right)-\Psi\left(q_{2} f\right), \Psi\left(h_{2}\right)-\Psi\left(q_{2} f\right), \ldots, \Psi\left(h_{n-1}\right)-\Psi\left(q_{2} f\right)\right\|_{\beta} .
\end{aligned}
$$


Since $\left\|r f-q_{2} f, h_{1}-q_{2} f, h_{2}-q_{2} f, \ldots, h_{n-1}-q_{2} f\right\|_{\alpha} \neq 0$,

$$
\left\|\Psi(f), \Psi\left(h_{1}\right)-\Psi\left(q_{2} f\right), \Psi\left(h_{2}\right)-\Psi\left(q_{2} f\right), \ldots, \Psi\left(h_{n-1}\right)-\Psi\left(q_{2} f\right)\right\|_{\beta} \neq 0 .
$$

Thus we have $r+q_{2}<q_{2}-q_{1}$, which is a contradiction. Hence $k=r$, that is, $\Psi(r f)=$ $r \Psi(f)$ for all positive real numbers $r$. Therefore $\Psi$ is $\mathbb{R}$-linear, as desired.

We get the following corollary from Theorems 4.1 and 4.2.

Corollary 4.1 Let $\Psi$ be an $n$-Lipschitz mapping with the $n$-Lipschitz constant $\kappa \leq 1$. Suppose that if $f, g, h$ are 2-collinear, then $\Psi(f), \Psi(g), \Psi(h)$ are 2-collinear. If $\Psi$ satisfies $(n \mathrm{DOPP})$, then $\Psi$ is an affine $n$-isometry.

\section{Conclusion}

In this article, the concept of 2-fuzzy $n$-normed linear space is defined and the concepts of $n$-isometry, $n$-collinearity, $n$-Lipschitz mapping are given. Also, the MazurUlam theorem is generalized into 2-fuzzy $n$-normed linear spaces.

\section{Acknowledgements}

The authors would like to thank the referees and area editor Professor Mohamed A. El-Gebeily for giving useful suggestions and comments for the improvement of this article.

\section{Author details}

${ }^{1}$ Department of Mathematics, Research Institute for Natural Sciences, Hanyang University, Seoul 133-791, Korea

${ }^{2}$ Department of Mathematics, Faculty of Science and Arts, Celal Bayar University, 45140 Manisa, Turkey

\section{Authors' contributions}

All authors conceived of the study, participated in its design and coordination, drafted the manuscript, participated in the sequence alignment, and read and approved the final manuscript.

\section{Competing interests}

The authors declare that they have no competing interests.

Received: 24 May 2011 Accepted: 19 January 2012 Published: 19 January 2012

\section{References}

1. Gähler, S: Lineare 2-normierte raume. Math Nachr. 28, 1-43 (1964). doi:10.1002/mana.19640280102

2. Gähler, S: Untersuchungen über verallgemeinerte m-metrische räume. I Math Nachr. 40, 165-189 (1969). doi:10.1002/ mana.19690400114

3. Misiak, A: $n$-inner product spaces. Math Nachr. 140, 299-319 (1989). doi:10.1002/mana.19891400121

4. Kim, SS, Cho, YJ: Strict convexity in linear n-normed spaces. Demonstratio Math. 29, 739-744 (1996)

5. Malčeski, R: Strong $n$-convex n-normed spaces. Mat Bilten. 21, 81-102 (1997)

6. Gunawan, H, Mashadi, M: On n-normed spaces. Intern J Math Math Sci. 27, 631-639 (2001). doi:10.1155/ S0161171201010675

7. Cheng, SC, Mordeson, JN: Fuzzy linear operators and fuzzy normed linear spaces. Bull Calcutta Math Soc. 86, 429-436 (1994)

8. Bag, T, Samanta, SK: Finite dimensional fuzzy normed linear spaces. J Fuzzy Math. 11, 687-705 (2003)

9. Narayanan, AL, Vijayabalaji, S: Fuzzy n-normed linear space. Intern J Math Math Sci. 24, 3963-3977 (2005)

10. Somasundaram, RM, Beaula, T: Some Aspects of 2-fuzzy 2-normed linear spaces. Bull Malays Math Sci Soc. 32, 211-221 (2009)

11. Mazur, S, Ulam, S: Sur les transformationes isométriques d'espaces vectoriels normés. CR Acad Sci Paris. 194, 946-948 (1932)

12. Baker, JA: Isometries in normed spaces. Am Math Monthly. 78, 655-658 (1971). doi:10.2307/2316577

13. Jian, W: On the generations of the Mazur-Ulam isometric theorem. J Math Anal Appl. 263, 510-521 (2001). doi:10.1006/ jmaa.2001.7627

14. Rassias, ThM, Wagner, P: Volume preserving mappings in the spirit of the Mazur-Ulam theorem. Aequationes Math. 66, 85-89 (2003). doi:10.1007/s00010-003-2669-7

15. Väisälä, J: A proof of the Mazur-Ulam theorem. Am Math Monthly. 110, 633-635 (2003). doi:10.2307/3647749

16. Chu, H: On the Mazur-Ulam problem in linear 2-normed spaces. J Math Anal Appl. 327, 1041-1045 (2007). doi:10.1016/j. jmaa.2006.04.053

17. Chu, H, Choi, S, Kang, D: Mappings of conservative distances in linear n-normed spaces. Nonlinear Anal. 70, 1168-1174 (2009). doi:10.1016/.na.2008.02.002

18. Rassias, ThM, Šemrl, P: On the Mazur-Ulam problem and the Aleksandrov problem for unit distance preserving mappings. Proc Amer Math Soc. 118, 919-925 (1993). doi:10.1090/S0002-9939-1993-1111437-6

19. Moslehian, MS, Sadeghi, Gh: A Mazur-Ulam theorem in non-Archimedean normed spaces. Nonlinear Anal. 69 3405-3408 (2008). doi:10.1016/.na.2007.09.023 
20. Choy, J, Chu, H, Ku, S: Characterizations on Mazur-Ulam theorem. Nonlinear Anal. 72, 1291-1297 (2010). doi:10.1016/j. na.2009.08.017

21. Choy, J, Ku, S: Characterization on 2-isometries in non-Archimedean 2-normed spaces. J Chungcheong Math Soc. 22, 65-71 (2009)

22. Chen, $X Y$, Song, MM: Characterizations on isometries in linear n-normed spaces. Nonlinear Anal. 72, 1895-1901 (2010). doi:10.1016/j.na.2009.09.029

23. Cobzaș, S: A Mazur-Ulam theorem for probabilistic normed spaces. Aequa-tiones Math. 77, 197-205 (2009). doi:10.1007/ s00010-008-2933-y

24. Alsina, C, Schweizer, B, Sklar, A: On the definition of a probabilistic normed space. Aequationes Math. 46, 91-98 (1993). doi:10.1007/BF01834000

25. Cho, YJ, Rahbarnia, F, Saadati, R, Sadeghi, Gh: Isometries in probabilistic 2-normed spaces. J Chungcheong Math Soc. 22, 623-634 (2009)

26. Alaca, C: A new perspective to the Mazur-Ulam problem in 2-fuzzy 2-normed linear spaces. Iranian J Fuzzy Syst. 7, 109-119 (2010)

27. Kang, D, Koh, H, Cho, IG: On the Mazur-Ulam theorem in non-Archimedean fuzzy normed spaces. App Math Lett. 25 301-304 (2012). doi:10.1016/j.aml.2011.09.004

28. Kubzdela, K: Isometries, Mazur-Ulam theorem and Aleksandrov problem for non-Archimedean normed spaces. Nonlinear Anal. (in press)

29. Rassias, ThM: On the A.D. Aleksandrov problem of conservative distances and the Mazur-Ulam theorem. Nonlinear Anal. 47, 2597-2608 (2001). doi:10.1016/50362-546X(01)00381-9

30. Xiang, S: Mappings of conservative distances and the Mazur-Ulam theorem. J Math Anal Appl. 254, 262-274 (2001). doi:10.1006/jmaa.2000.7276

31. Cho, YJ, Lin, PCS, Kim, SS, Misiak, A: Theory of 2-Inner Product Spaces. Nova Science Publishers, New York (2001)

doi:10.1186/1029-242X-2012-14

Cite this article as: Park and Alaca: An introduction to 2-fuzzy $n$-normed linear spaces and a new perspective to the Mazur-Ulam problem. Journal of Inequalities and Applications 2012 2012:14.

\section{Submit your manuscript to a SpringerOpen ${ }^{\odot}$} journal and benefit from:

- Convenient online submission

- Rigorous peer review

- Immediate publication on acceptance

- Open access: articles freely available online

- High visibility within the field

- Retaining the copyright to your article

Submit your next manuscript at $\gg$ springeropen.com 